\title{
IS ONLINE STROKE-SPECIFC EDUCATION SUITABLE, ACCEPTABLE, AND EFFECTIVE FOR THE CONTINUED PROFESSIONAL DEVELOPMENT OF STROKE UNIT STAFF? AN EVALUATION OF THE STROKE-FACTS ONLINE EDUCATIONAL RESOURCE \\ Colette Miller, Eleanor Briggs \& Professor Dame Caroline Watkins University of Central Lancashire, Stroke Research Team, School of Nursing, Faculty of Health and Wellbeing
}

\section{Introduction}

Why is stroke-specific training important?

- $>100,000$ strokes occur each year in the UK ${ }^{1}$

- Care on acute specialised stroke units (ASSUs) can improve patient outcomes ${ }^{2}$

- Undergraduate courses may not deliver sufficient strokespecific knowledge required for effective ASSU care $^{3}$

- $30 \%$ of ASSU staff had no stroke-specific training ${ }^{4}$

How is stroke-specific training standardised?

- The Department of Health funded Stroke-Specific Education Framework ${ }^{5}$ (SSEF)

- Outlines stroke-specific knowledge required for various healthcare professional (HCP) roles and allows HCPs to self assess their knowledge, identify unmet learning needs, and locate relevant training

What stroke-specific training already exists?

- Stroke-FACTS: supplements undergraduate curriculum, bridging the gap between graduate and newly qualified

- Based on mapping of content of 9 multidisciplinary HCP undergraduate courses

- Aligned to SSEF standardised HCP role profiles

- Content aimed at SSEF knowledge level 2; Factual Knowledge (newly qualified)

\section{Research Aim}

- To evaluate the suitability, acceptability, and effectiveness of Stroke-FACTS for the education of practising HCPs

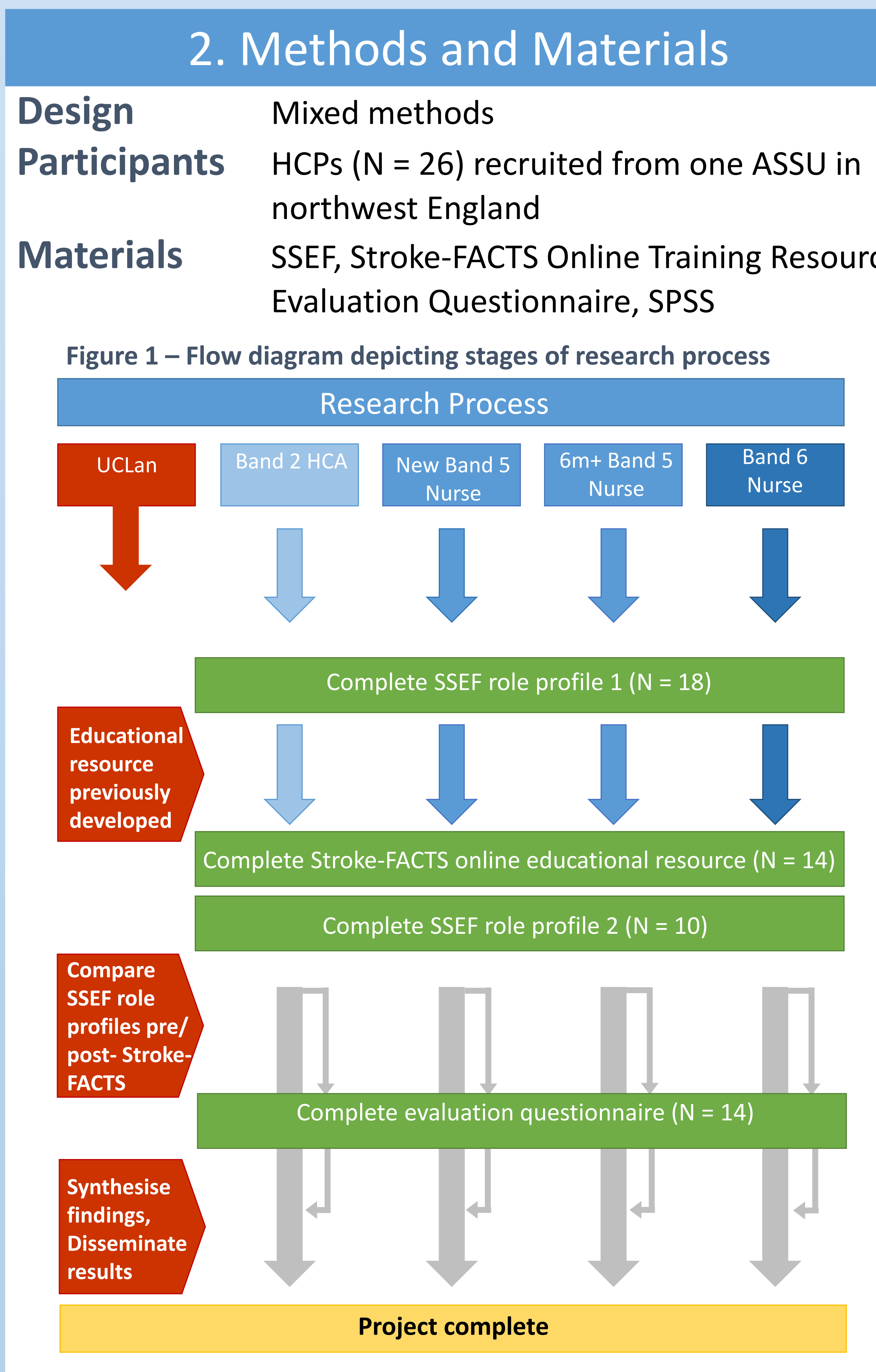

0

0

\section{References}

'STROKE ASSOCIATION, 2017. State of the nation: stroke statistics

2STROKE UNIT TRIALISTS' COLLABORATION, S. U. 2013. Organised inpatient (stroke unit) care for stroke. Cochrane Database Syst Rev, 9 .

${ }^{3}$ MASON-WHITEHEAD, E., RIDGWAY, V. \& BARTON, J. 2013. Passed without a stroke: A UK mixed method study exploring student nurses' knowledge of stroke. Nurse education today, $33,998-1002$.

${ }^{4}$ SMITH, L. N., CRAIG, L. E., WEIR, C. J. \& MCALPINE, C. H. 2008. Stroke education for healthcare professionals: making it fit for PSMITH, L. N., CRAIG, L. E., WEIR, C.J. \& MCALP
purpose. Nurse education today, $28,337-347$.

${ }^{5}$ WATKINS C, BARRON T, DAVIS D, HATTON S, JENKINSON D, JONES S, PRICE C, SOUTH A, QUINN T, LEATHLEY M. The UK forum for stroke training and the stroke-specific education framework. Journal of Paramedic Practice. 2011 Sep 2;3(9):481-2.

Suitable?

Effective?

Conclusions
Statistical analysis of pre and post-training knowledge scores

Of the $10 \mathrm{HCPs}$ (Nurses $\mathrm{N}=6$; Healthcare Assistants $\mathrm{N}=4$ ) who completed all three stages of the research, $90 \%$ had unmet learning needs according to the SSEF. One had none and was discounted from analysis

A Wilcoxon signed-rank test revealed a statistically significant increase in knowledge following Stroke-FACTS online training, $Z=-2.023, p<.043$, with a medium effect size $(r=.45)$

The median \% knowledge match to the SSEF increased from $(M d=81)$ pre-Stroke-FACTS to $(M d=87)$

\section{Qualitative Data}

HCPs perceptions regarding the training resource were explored using a free text questionnaire. Thematic analysis revealed 5 themes outlined below:

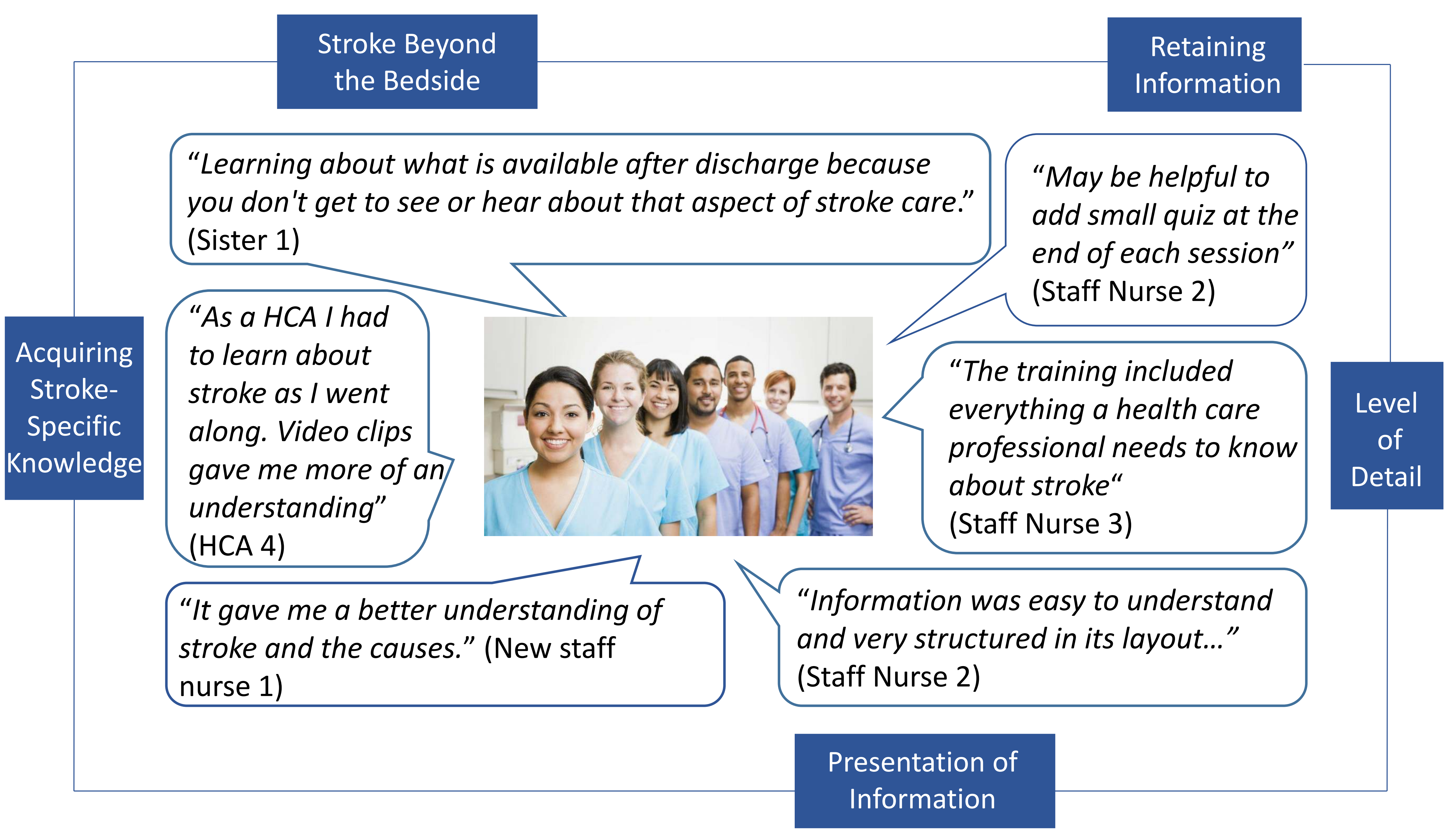

Questionnaire data detailing health care professionals' opinions of Stroke-FACTS

Acceptable?

$100 \%$ of HCPs stated that they would recommend Stroke-FACTS to a colleague

92\% of HCPs felt Stroke-FACTS was a suitable resource for use by practising HCPs

$100 \%$ of HCPs felt Stroke-FACTS provided new information, beyond their existing

knowledge: $54 \%$ = some new information; $46 \%$ = lots of new information

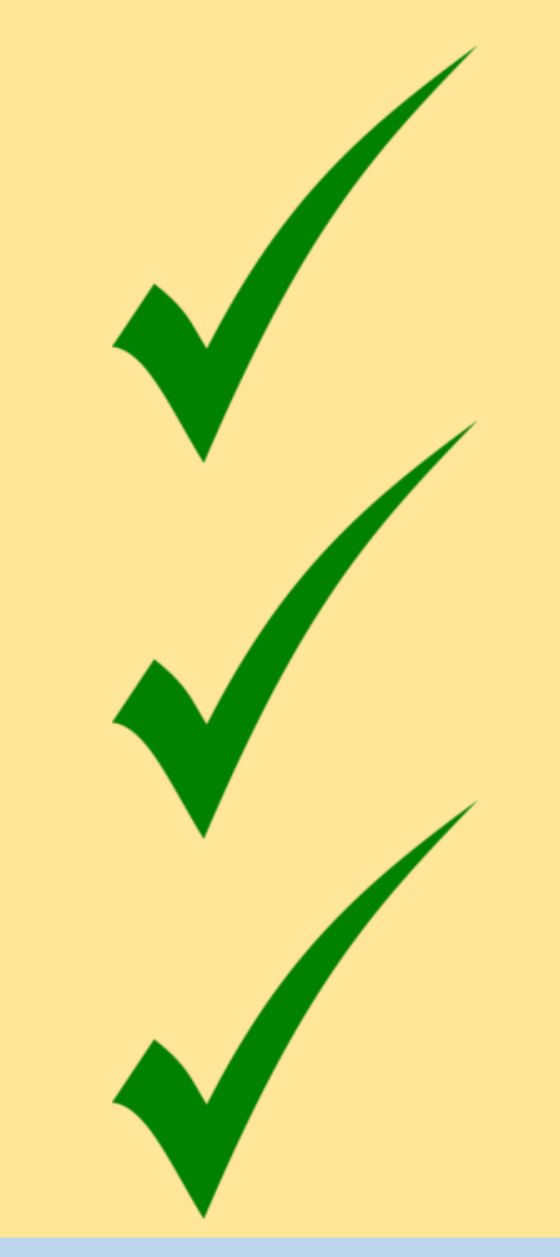

\section{Conclusion}

- The findings support previous research suggesting that HCPs have unmet stroke-specific learning needs

- Access to educational resources, such as Stroke-FACTS, can potentially increase knowledge

- Utilising frameworks to identify learning needs, and develop educational resources, can contribute to the provision of clinically relevant teaching

Further Research

- Refine Stroke-FACTS educational resource in response to HCP feedback

- Explore the facilitators and barriers to training completion for HCPS

- Investigate whether completion of Stroke-FACTS improves patient outcomes

\section{For information}

- To find out more about the Stroke-Specific Education Framework visit: www.stroke-education.org.uk or email SSEFEnquiries@uclan.ac.uk

- Enquiries regarding this research should be directed to $\underline{\text { cmiller5@uclan.ac.uk }}$

S. Stroke-Specific Education
(E) Framework

